\title{
Revised absolute amplitude calibration of the LOPES experiment
}

\author{
K. Link ${ }^{1}$, T. Huege ${ }^{* 2}$, W.D. Apel ${ }^{2}$, J.C. Arteaga-Velázquez ${ }^{3}$, L. Bähren ${ }^{4}$, K. Bekk ${ }^{2}$, \\ M. Bertaina ${ }^{5}$, P.L. Biermann ${ }^{5,1}$, J. Blümer ${ }^{1,6}$, H. Bozdog ${ }^{2}$, I.M. Brancus ${ }^{7}$, \\ E. Cantoni ${ }^{4,8}$, A. Chiavassa ${ }^{5}$, K. Daumiller ${ }^{2}$, V. de Souza ${ }^{9}$, F. Di Pierro ${ }^{5}$, P. Doll ${ }^{2}$, \\ R. Engel ${ }^{2}$, H. Falcke ${ }^{10,3,5}$, B. Fuchs ${ }^{1}$, H. Gemmeke ${ }^{11}$, C. Grupen $^{12}$, A. Haungs ${ }^{2}$, \\ D. Heck ${ }^{2}$, R. Hiller ${ }^{2}$, J.R. Hörandel ${ }^{10}$, A. Horneffer ${ }^{6}$, D. Huber ${ }^{1}$, P.G. Isar ${ }^{13}$, \\ K-H. Kampert ${ }^{14}$, D. Kang ${ }^{1}$, O. Krömer ${ }^{11}$, J. Kuijpers ${ }^{10}$, P. Łuczak ${ }^{15}$, M. Ludwig ${ }^{1}$, \\ H.J. Mathes ${ }^{2}$, M. Melissas ${ }^{1}$, C. Morello ${ }^{8}$, J. Oehlschläger ${ }^{2}$, N. Palmieri ${ }^{1}$, T. Pierog ${ }^{2}$, \\ J. Rautenberg ${ }^{14}$, H. Rebel ${ }^{2}$, M. Roth ${ }^{2}$, C. Rühle ${ }^{11}$, A. Saftoiu ${ }^{7}$, H. Schieler ${ }^{2}$, \\ A. Schmidt ${ }^{11}$, S. Schoo ${ }^{2}$, F.G. Schröder ${ }^{2}$, O. Sima ${ }^{16}$, G. Toma ${ }^{7}$, G.C. Trinchero ${ }^{8}$, \\ A. Weindl ${ }^{2}$, J. Wochele ${ }^{2}$, J. Zabierowski ${ }^{15}$, J.A. Zensus ${ }^{5}$ - LOPES Collaboration \\ ${ }^{1}$ Institut für Experimentelle Kernphysik, Karlsruher Institut für Technologie (KIT), Germany \\ ${ }^{2}$ Institut für Kernphysik, Karlsruher Institut für Technologie (KIT), Germany \\ ${ }^{3}$ Instituto de Física y Matemáticas, Universidad Michoacana, Morelia, Mexico \\ ${ }^{4}$ ASTRON, Dwingeloo, The Netherlands \\ ${ }^{5}$ Dipartimento di Fisica, Università degli Studi di Torino, Torino, Italy \\ ${ }^{6}$ Max-Planck-Institut für Radioastronomie, Bonn, Germany \\ ${ }^{7}$ National Institute of Physics and Nuclear Engineering, Bucharest-Magurele, Romania \\ ${ }^{8}$ Osservatorio Astrofisico di Torino, INAF Torino, Italy \\ ${ }^{9}$ Universidade São Paulo, Instituto de Física de São Carlos, São Carlos, Brasil \\ ${ }^{10}$ Department of Astrophysics, Radboud University Nijmegen, The Netherlands \\ ${ }^{11}$ Institut für Prozessdatenverarbeitung und Elektronik, KIT, Germany \\ ${ }^{12}$ Faculty of Natural Sciences and Engineering, Universität Siegen, Germany \\ ${ }^{13}$ Institute for Space Sciences, Bucharest-Magurele, Romania \\ ${ }^{14}$ Fachbereich C, Physik, Universität Wuppertal, Germany \\ 15 Department of Astrophysics, National Centre for Nuclear Research, Eódź, Poland \\ ${ }^{16}$ Department of Physics, University of Bucharest, Bucharest, Romania
}

E-mail: tim.huege@kit.edu

The 34th International Cosmic Ray Conference,

30 July- 6 August, 2015

The Hague, The Netherlands

\footnotetext{
* Speaker.
} 


\begin{abstract}
One of the main aims of the LOPES experiment was the evaluation of the absolute amplitude of the radio signal of air showers. This is of special interest since the radio technique offers the possibility for an independent and highly precise determination of the energy scale of cosmic rays on the basis of signal predictions from Monte Carlo simulations. For the calibration of the amplitude measured by LOPES we used an external source. Previous comparisons of LOPES measurements and simulations of the radio signal amplitude predicted by CoREAS revealed a discrepancy of the order of a factor of two. A re-measurement of the reference calibration source, now performed for the free field, was recently performed by the manufacturer. The updated calibration values lead to a lowering of the reconstructed electric field measured by LOPES by a factor of $2.6 \pm 0.2$ and therefore to a significantly better agreement with CoREAS simulations. We discuss the updated calibration and its impact on the LOPES analysis results.
\end{abstract}




\section{Introduction}

In the past decade, tremendous progress was made in the understanding of radio emission from extensive air showers and the reconstruction of cosmic ray properties from radio measurements [1]. One of the most challenging aspects of radio detection of extensive air showers turned out to be the absolute calibration of the detectors. In the historical experiments, there were in fact strong disagreements between results from different experiments, likely due to calibration issues [2].

In LOPES [3], we have made considerable effort from the very beginning of the experiment to provide a high-quality absolute calibration [4]. Furthermore, we compared our measurements with predictions from Monte Carlo simulations with REAS3.11 [5] and CoREAS [6]. These simulation codes calculate the radio emission from an extensive air shower from first principles (movement of charged particles plus classical electrodynamics using the endpoint formalism [7]). Given a set of parameters for the primary cosmic ray, the Monte Carlo simulation of the extensive air shower fully determines the result. The simulations contain no free parameters that could be tuned and thus provide an absolute prediction of the expected radio amplitude. This is very important, because such simulations can thus be used to calibrate the energy scale of cosmic ray detectors.

In a previous analysis [8] we concluded that LOPES data were in agreement with predictions from the (simplified and thus obsolete) REAS3.11 simulation code, but were discrepant with predictions of the (more advanced and thus more precise) CoREAS simulation code. The measured amplitudes were approximately a factor of two larger than those predicted by CoREAS. We have investigated possible causes for this discrepancy and have ruled out problems in the analysis procedure and the CoREAS simulation code. It turned out, however, that the calibration of the LOPES antennas using an external reference source was based on unsuited calibration data for the reference source. In the following we describe how the calibration was updated, then illustrate the effects of the updated calibration on parameters derived from a data set of cosmic ray showers measured with LOPES, and finally present an updated comparison of LOPES data with CoREAS simulations.

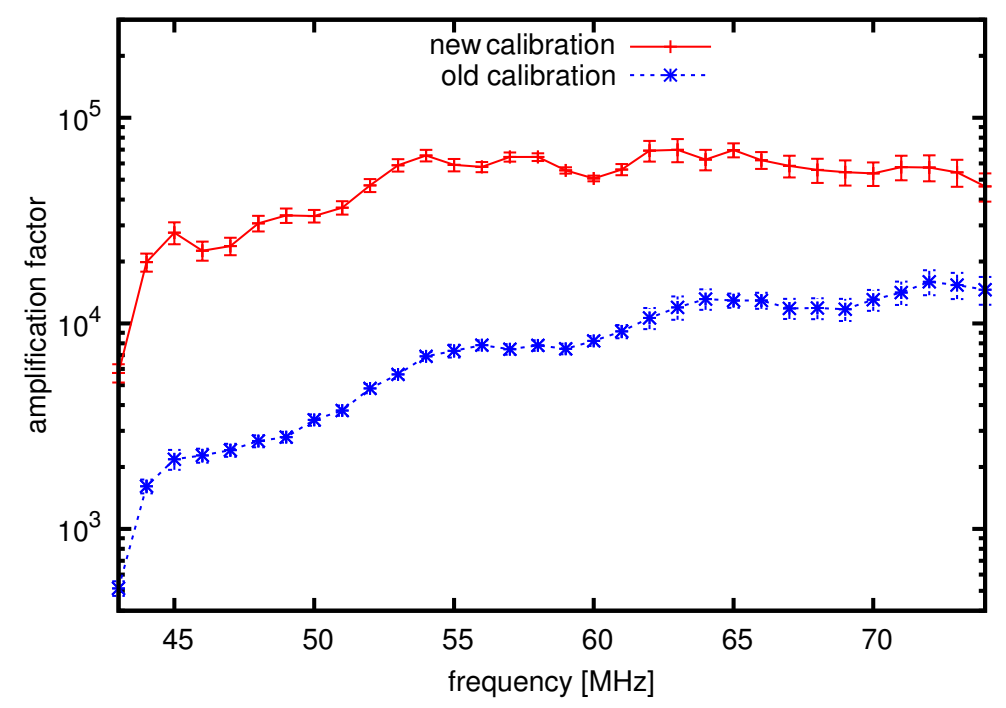

Figure 1: Comparison of the amplification factors (related to power) of the analog chain of a LOPES antenna based on old and revised calibration data for the reference source. The effect is the same for all antennas. 

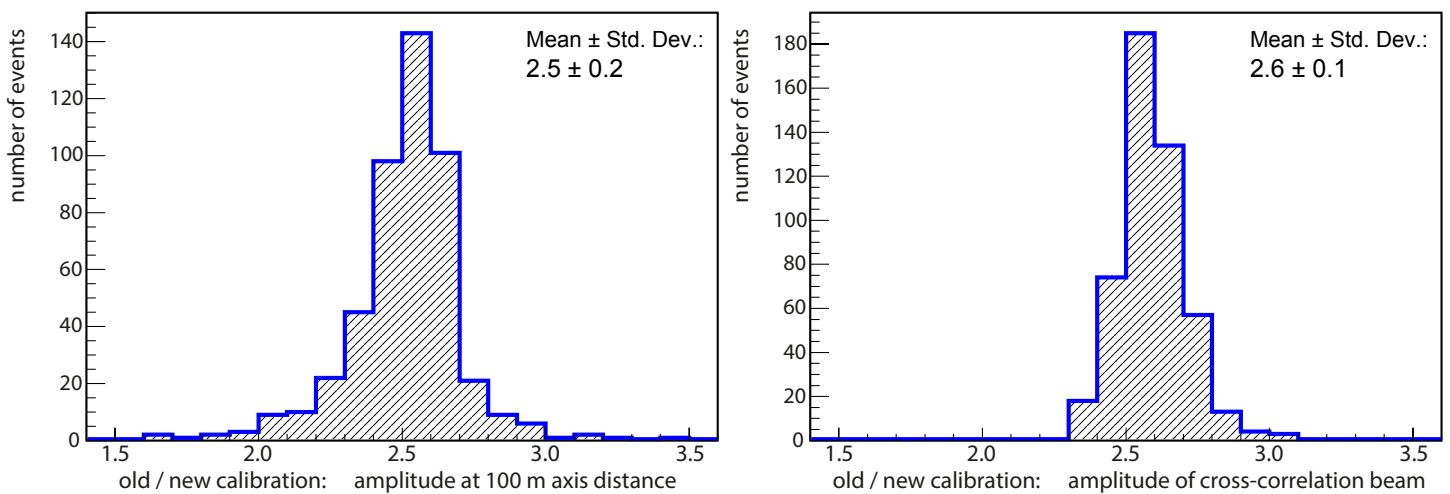

Figure 2: Influence of the revised calibration on the amplitude at $100 \mathrm{~m}$ axis distance $\varepsilon_{100}$ (left) and on the amplitude of the cross-correlation beam (right) as measured with LOPES.

\section{Revised Amplitude Calibration}

The calibration of LOPES uses an external reference source [4], for which the manufacturer provided the absolute power radiated as a function of frequency. We deployed the calibration source $10 \mathrm{~m}$ above the LOPES antennas, measured the power received in the LOPES antennas as a function of frequency, and then derived the amplification factors of our analog chain as a function of frequency [4].

We performed an independent re-implementation of the analysis part of the calibration procedure, finding no problem that could explain the observed discrepancies between LOPES data and CoREAS simulations. Hence, we contacted the manufacturer of the calibration source to verify the validity of the provided calibration data. In detailed discussions it was realized that the calibration data we had originally received were acquired in a horizontal measurement setup with a reflective ground (free-field conditions). However, the measurement of air showers by LOPES is better described with free-space conditions. Therefore, we requested a re-calibration of the reference source and received updated calibration values from the manufacturer for free-space conditions.

In Fig. 1 we demonstrate the difference between the amplification factors for our analog chain as derived using the old and new calibration data. The new values yield amplification factors that are systematically higher than those derived with the original calibration data. The same correction applies to all antennas of the LOPES array, as the same reference source was used to calibrate all LOPES antennas. There is, however, a slight dependence of the correction between old and new calibration on frequency. We thus investigate in a detailed study how the updated calibration values influence the LOPES event reconstruction. We note that the systematic uncertainty on the absolute calibration of the LOPES amplitude scale previously reported as 35\% constituted the two-sigma uncertainty provided by the manufacturer of the reference source. The one-sigma uncertainty for the revised absolute amplitude calibration amounts to $16 \%$.

\section{Influence of the new calibration}

We analyzed LOPES data acquired between the end of 2005 and the end of 2009 with an energy reconstructed from KASCADE-Grande $[9,10]$ data above $10^{17} \mathrm{eV}$, a zenith angle below 

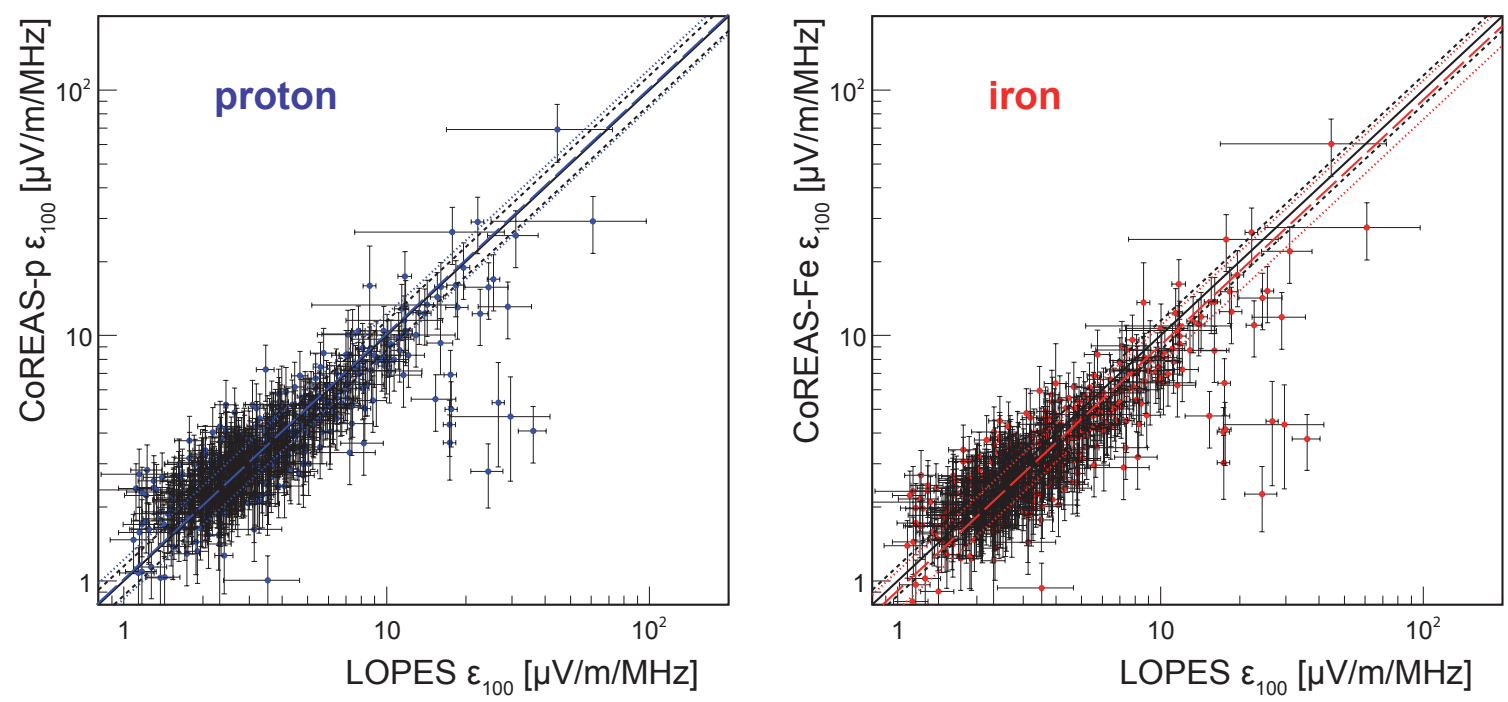

Figure 3: Per-event comparison of $\varepsilon_{100}$ derived from LOPES measurements and from CoREAS simulations for a simulation set of proton-induced showers (left) and iron-induced showers (right). The black lines indicate the 1:1 expectation (solid) and the systematic scale uncertainty of the amplitude calibration corresponding to $16 \%$ (dashed). The colored lines mark the actual correlation between simulations and data (long-dashed) and the associated systematic uncertainty of the predicted amplitudes of $20 \%$ arising from the energy reconstruction of KASCADE-Grande (dotted).

$45^{\circ}$, shower core inside the KASCADE or Grande fiducial areas, and standard KASCADE-Grande cuts. Thunderstorm events are excluded. The events are required to have a clear radio signal which is selected via the cross-correlation beam, i.e., the correlated power must be larger than $80 \%$ of the full power, and the signal-to-noise ratio must be larger than 14 , normalized with a factor $\sqrt{N_{\text {ant }} / 30}$ to take into account the number of antennas available for a particular measurement. With these conditions approximately 500 events remain for the analysis. In addition to the revised calibration, we made small improvements in our analysis, including an update of the antenna model to more realistic ground conditions, and an improved fitting algorithm for the lateral distribution function.

We perform our standard analysis procedure on the LOPES data, which identifies the pulses in individual antennas, quantifies their pulse amplitudes and fits an exponential lateral distribution to the measured radio amplitude $\varepsilon$ as a function of axis distance $d$ :

$$
\varepsilon(d)=\varepsilon_{100} \exp [-\eta(d-100 \mathrm{~m})]
$$

The two parameters derived from this fit are the amplitude at a distance of $100 \mathrm{~m}$ from the shower axis, $\varepsilon_{100}$, and the slope parameter characterizing the steepness of the lateral distribution, $\eta$. The parameter $\varepsilon_{100}$ is an estimator for the cosmic ray energy [11], and the slope parameter $\eta$ can be related to the depth of shower maximum [12].

In Fig. 2 we illustrate the change of $\varepsilon_{100}$ and the amplitude of the cross-correlation beam with respect to the LOPES data presented in [8]. On average, the amplitude drops by a factor of $2.6 \pm 0.2$ when switching to the revised calibration, where the factor is the same within uncertainties for $\varepsilon_{100}$ and the amplitude of the cross-correlation beam. This change is purely due to the new calibration, the minor improvements in the analysis pipeline had no significant effect on the amplitude scale. 

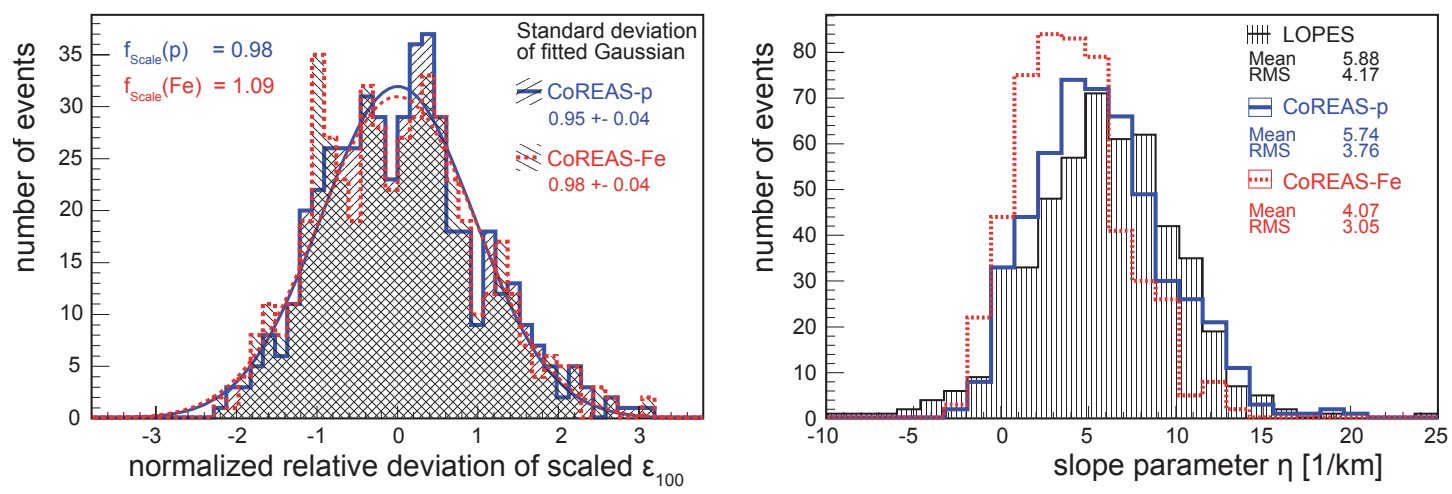

Figure 4: Left: Histogram of the per-event deviations of $\varepsilon_{100}$ between LOPES data and CoREAS simulations normalized by the per-event uncertainty in $\varepsilon_{100}$. The mean offset factors between simulations and data of 0.98 for proton simulations and 1.09 for iron simulations have been corrected before histogramming (to obtain distributions with a mean of zero). Right: Comparison of the $\eta$ distributions derived from LOPES data and CoREAS simulations.

The slope parameter $\eta$ was affected by both the minor analysis improvements and the revised calibration, but changes were within the method-related systematic uncertainty of approximately $1 / \mathrm{km}$ and in fact compensated each other (see next section).

\section{Comparison of data and CoREAS simulations}

In a next step, we repeat the comparison of LOPES data with CoREAS simulations. We perform the same analysis as presented in [8]. This means that for each LOPES event we compare with one CoREAS simulation of a proton-induced air shower and one CoREAS simulation of an iron-induced air shower. The peak amplitudes for the individual simulated antennas are determined from the bandpass-filtered simulations output. (The influence of a complete detector simulation has also been investigated and is presented in another contribution at this conference[13].) The simulated peak amplitudes are used as input for the same lateral distribution fit as applied to the LOPES data. Afterwards, the parameters $\varepsilon_{100}$ and $\eta$ as derived for LOPES data and CoREAS simulations are compared.

In Fig. 3, we compare $\varepsilon_{100}$ as measured with LOPES and simulated with CoREAS for each individual shower. There is good agreement between the simulated and measured data, well within the $16 \%$ systematic uncertainty on the absolute amplitude scale of the calibration. Previously, this comparison yielded a clear discrepancy between the CoREAS simulations and LOPES data [8]. (The few outliers were already present in the previous analysis; the reason for those is unknown.)

A more quantitative view of these data is given in Fig. 4 (left). The histogram shows the deviation between the measured and simulated $\varepsilon_{100}$ after correcting for the mean $\varepsilon_{100}$ offset factors of the proton and iron data sets. The mean offset factor for the proton simulations is 0.98 , i.e. the mean deviation between $\varepsilon_{100}$ values predicted by CoREAS and measured by LOPES in only $2 \%$ for proton, and $9 \%$ for iron simulations. This agreement is well within the systematic uncertainty of the amplitude calibration that the manufacturer of the reference source quantifies as $16 \%$. The width 


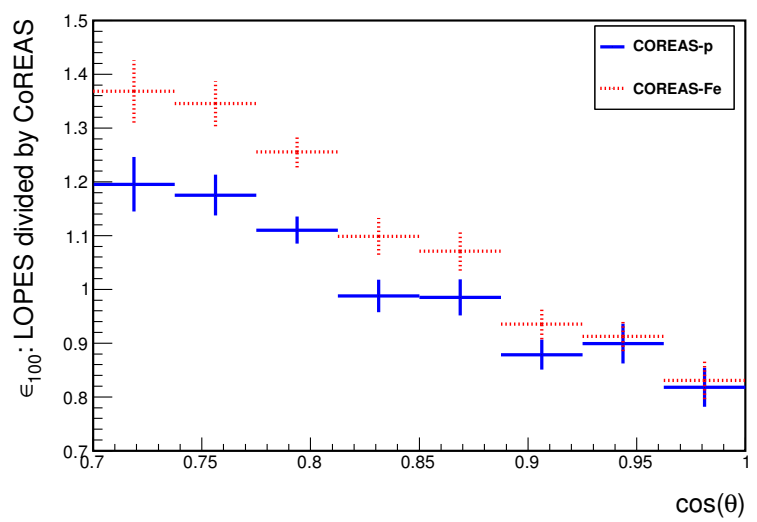

Figure 5: Offset factor for $\varepsilon_{100}$ between LOPES data and CoREAS simulations as a function of air shower zenith angle.

of the distributions correspond to one standard deviation, i.e., the distribution of the deviations conforms to the expectations from statistical uncertainties.

For the comparison of $\eta$, we do not directly compare individual measured showers and simulations, as the depth of shower maximum of each shower is unknown but influences $\eta$. Thus, we compare the $\eta$-distribution derived from data with those derived from the CoREAS simulation sets for proton- and iron-induced showers in Fig. 4 (right). The distributions agree well between simulations and data, as was already the case in the previous comparison [8]. The mean of the measured distribution is practically unchanged from the previously published result, well within the systematic uncertainty of approximately $1 / \mathrm{km}$ on $\eta$.

In a recent publication [14] we noted that there is a slight discrepancy in the amplitude scaling with zenith angle as predicted by CoREAS simulations and LOPES data. We investigated whether this discrepancy was removed by the revised calibration or the minor analysis improvements. As can be seen in Fig. 5, the discrepancy remains. The deviation is of the order that can be expected from uncertainties in the antenna directivity pattern and thus does not necessarily constitute a problem. Nevertheless, future analyses should pay attention to the zenith-dependence of the radio emission in comparison with predictions from simulation codes.

\section{Influence on published results}

The revised calibration directly influences the absolute amplitudes measured with LOPES, which on average turn out to be a factor of $2.6 \pm 0.2$ lower than those determined with the old calibration. This correction factor can be applied to all amplitude values previously published by LOPES, in particular any energy correlation results $[11,14]$. Our reanalysis demonstrated that no other relevant reconstruction quantities are affected significantly by the updated recalibration in conjunction with analysis improvements. In particular, results on the lateral slope $\eta$ remain valid.

\section{Conclusion}

Previously, LOPES had reported a discrepancy between measured amplitudes for radio emis- 
sion from extensive air showers and amplitudes predicted by state-of-the-art simulations with the CoREAS simulation code. A thorough investigation revealed that unsuitable calibration values had been used in the previous calibration of LOPES. New calibration values, suitable for free-space conditions, have been acquired from the manufacturer of the reference source. A reanalysis with the new calibration reveals that on average, amplitudes measured with LOPES drop by a factor of $2.6 \pm 0.2$ with respect to the old calibration. The slope of the lateral distribution of the radio signal is not affected significantly by the revised calibration in conjunction with minor analysis improvements.

With the new calibration, LOPES data and CoREAS simulations are in very good agreement, well within the $16 \%$ systematic uncertainty of the absolute amplitude scale of the calibration source. The only remaining issue is a slight discrepancy between the zenith-angle dependence of the electric field amplitudes observed in LOPES data and CoREAS simulations, which could, however, be explained by the uncertainties of the antenna directivity pattern. The (obsolete) REAS3.11 simulations are no longer in agreement with the LOPES data within systematic uncertainties using the revised calibration.

More precise tests of the absolute amplitude predictions of state-of-the-art simulation codes require experimental data with a smaller systematic scale uncertainty. Direct comparisons between Tunka-Rex [15], LOFAR [16] and LOPES data, however, will not be limited by the $16 \%$ scale uncertainty, as all three experiments have been cross-calibrated with the LOPES reference source, including a cross-check with Galactic noise [17].

\section{References}

[1] T. Huege: Braz. J. Phys. 44 (2014) 520

[2] V.B. Atrashkevich, et al.: Sov. J. Nucl. Phys. 28 (1978) 366

[3] H. Falcke et al. (LOPES Coll.): Nature 435 (2005) 313

[4] S. Nehls, et al.: Nucl. Instr. Meth. A 589 (2008) 350

[5] M. Ludwig, T. Huege: Astropart. Phys. 34 (2011) 438

[6] T. Huege, C.W. James, M. Ludwig: AIP Conf. Proc. 1535 (2013) 128

[7] C.W. James, et al.: Phys. Rev. E. 84 (2011) 056602

[8] W.D. Apel et al. (LOPES Coll.): Astropart. Phys. 50-52 (2013) 76

[9] T. Antoni et al. (KASCADE Coll.): Nucl. Instr. Meth. A 513 (2003) 490

[10] W.D. Apel et al. (KASCADE-Grande Coll.): Nucl. Instr. Meth. A 620 (2010) 202

[11] F.G. Schröder et al. (LOPES Coll.): Proc. of ECRS 2014, Kiel, Germany (2014), in press

[12] W.D. Apel et al. (LOPES Coll.): Phys. Rev. D 85 (2012) 071101(R)

[13] F.G. Schröder, et al. (LOPES Coll.): this issue (ICRC 2015) 317

[14] W.D. Apel et al. (LOPES Coll.): Phys. Rev. D 90 (2014) 062001

[15] P.A. Bezyazeekov, et al. (Tunka-Rex Coll.): Nucl. Instr. Meth. A, submitted

[16] P. Schellart, et al.: Astron. Astroph. 560 (2013) A98

[17] A. Nelles, et al: JINST, submitted, arXiv:1507.08932 